\title{
Histoire du thymus
}

D'un organe vestigial

\section{à la programmation}

de la tolérance

immunitaire

> Pendant des siècles, le thymus a été considéré comme un organe vestigial, et son rôle dans la différenciation des lymphocytes T n'a été proposé que dans les années 1960. Les plus récentes recherches lui attribuent un rôle unique dans la programmation de la tolérance immunitaire centrale. Le mécanisme en est la transcription dans l'épithélium thymique de gènes codant des précurseurs d'antigènes du soi. Leur apprêtement aboutit à la présentation d'antigènes du soi par le complexe majeur d'histocompatibilité (CMH) exprimé par les cellules épithéliales et dendritiques thymiques. Pendant la vie fœtale, cette présentation permet la sélection négative des clones de lymphocytes T portant des récepteurs (TCR) de haute affinité pour les complexes $\mathrm{CMH}$ /antigène du soi. Après la naissance, cette présentation promeut, de plus, la sélection de lymphocytes T régulateurs spécifiques de ces complexes. De nombreuses études, de même que l'identification des gènes Aire et Fezf2, ont montré qu'une défaillance du thymus joue un rôle crucial dans le développement de l'auto-immunité spécifique d'organes. <

\section{Le grand saut de l'antiquité au début du XXe siècle}

Le nom de thymus apparaît clairement dans les écrits de Galien (129-230 après JC). Ce médecin grec, né à Pergame puis installé à Rome, est demeuré une référence jusqu'au Siècle des Lumières. II considérait le thymus comme une glande dont la seule et simple fonction était d'établir une protection entre le sternum et la veine cave, une interprétation qui sera d'ailleurs reprise quinze siècles plus tard par Vésale (voir Encart pour une analyse historique approfondie de l'origine du mot thymus). Galien observa que le thymus était

Cet article est un résumé succinct de l'essai «Voyage[s] à travers le thymus » publié par l'auteur en septembre 2016 par les Presses Universitaires de Liège.

plus gros chez le jeune animal et diminuait avec l'âge. Jacopo Berengario de Carpi (1460-1530) fut le premier anatomiste à découvrir le thymus humain en disséquant des cadavres à l'université de Bologne, un des grands centres européens de l'anatomie à l'époque, avec Padoue et Paris. II décrivit avec précision tant la vascularisation que l'innervation du thymus.

Avec l'aide des élèves du peintre italien Le Titien (1489-1576) à Venise, le Belge André Vésale (1514-1564), le grand anatomiste de la Renaissance, publia à Padoue la première planche anatomique montrant le thymus dans De Humani Corporis Fabrica : un petit organe multilobé situé juste en-dessous du sternum que Vésale interprétait aussi comme un coussinet protecteur de la vascularisation du médiastin supérieur. Bartolomeo Eustachi (1510-1574), qui a décrit les glandes surrénales, dessina également le thymus en montrant ses rapports anatomiques dans le médiastin antérieur.

À la même époque, le chirurgien Ambroise Paré (1510-1590) le mentionne comme une glande fort molle et de consistance spongieuse et Félix Plater (1536-1614), un médecin suisse, rapporte un cas de mort par asphyxie d'un nourrisson, attribué à la compression de sa trachée par une masse interne développée à partir du thymus.

Au siècle suivant, le médecin anglais Francis Glisson (1599-1677), qui décrit le rachitisme des enfants, émet l'hypothèse que le thymus produit un fluide destiné à l'alimentation et à la croissance du nourrisson. William Hewson (1739-1774), chirurgien anglais connu pour sa découverte de la fibrine, publie le premier traité sur le thymus : il rapporte aussi ses variations de taille avec l'âge, son involution en cas 
de maladies aiguës ou chroniques, et note que le thymus est rempli de «particules » semblables à celles que l'on retrouve dans le sang et dans la lymphe. Il en déduit que le thymus n'existe qu'au début de la vie lorsque ces particules semblent les plus nécessaires.

En 1832, Astley Paston Cooper (1768-1841) publie The Anatomy of the Thymus Gland riche en illustrations précises du thymus. II décrit aussi un cancer du thymus, le thymome malin. Plus tard, un essai sur la physiologie de la glande thymique (1845) vaut au chirurgien anglais John Simon (1816-1904) sa nomination à la Royal Society of London et Arthur Hill Hassall (1817-1894), médecin-chimiste britannique, compare la structure histologique du thymus avec celle d'autres organes lymphatiques (rate et ganglions) et décrit les corpuscules qui portent aujourd'hui son nom dans la zone médullaire du thymus ${ }^{1}$.

L'embryologiste écossais John Beard (1858-1924) considèrera ensuite que le thymus pourrait être la source de toutes les cellules lymphocytaires de l'organisme. Il suggéra, par ailleurs, une analogie de pouvoir invasif entre cancer et trophoblaste placentaire. En effet, selon lui, le cancer serait un trophoblaste irresponsable. En 1902, il publiera d'ailleurs, dans le Lancet, l'article intitulé Embryological aspects and etiology of carcinoma qui préfigure le concept actuel des cellules souches cancéreuses. Il est nominé en 1906 pour le prix Nobel de physiologie ou médecine. Sa nomination était ainsi argumentée : «Pour la découverte de l'existence chez les premiers vertébrés d'une structure nerveuse qui ne se développe et qui n'est fonctionnelle que pendant les stades embryonnaires précoces, la découverte de la vraie nature de la glande thymique, et la démonstration de la continuité morphologique directe des cellules germinatives de tous les vertébrés ». Mais le prix Nobel sera décerné en définitive à Camillo Golgi et Santiago Ramon y Cajal².

\section{Le thymus, entre endocrinologie et immunologie}

Au début du XXe siècle, on parle de «l'énigme du thymus ». II pourrait s'agir d'une glande de nature épithéliale infiltrée par de nombreux petits lymphocytes au second mois de la vie embryonnaire chez I'homme. Ces lymphocytes intra-thymiques se divisent beaucoup. Contrairement aux cellules épithéliales de la trame thymique, ils sont par conséquent très sensibles aux rayons Röntgen ${ }^{3}$ récemment découverts. La communauté scientifique pense alors que le thymus est un organe «vestigial », transitoire, qui décroît précocement et cesse de fonctionner très tôt dans la vie. Pourtant dès 1890, l'anatomiste allemand Wilhelm Waldeyer (1836-1921) remarque que, chez des personnes âgées, subsiste un thymus graisseux rempli d'îlots résiduels de tissu. Jan-August Hammar (Suède, 1861-1946) le confirme en 1906 et précise que, si le thymus atteint son développement maximal au moment de la puberté, du tissu thymique normal persiste jusqu'à un âge avancé. Chez les animaux, il montre que la castration avant la puberté assure le maintien d'un volume important de tissu thymique

\footnotetext{
${ }^{1}$ Les corpuscules de Hassall sont des îlots de cellules épithéliales pavimenteuses kératinisées, hyalinisées, parfois kystiques, situées dans la médullaire du thymus.

${ }^{2}$ Camillo Golgi et Santiago Ramon y Cajal ont reçu le prix Nobel de physiologie ou médecine en 1906, pour leurs études de la structure du système nerveux.

${ }^{3}$ Les rayons $X$ que Wilhelm Röntgen a découverts en 1895 .
}

et qu'une involution du thymus accompagne les états de grossesse et de dénutrition, de même que des maladies infectieuses. À l'inverse, il observe une hyperplasie du thymus dans l'hyperthyroïdie de la maladie de Basedow (une maladie auto-immune de la thyroïde), l'insuffisance surrénalienne d'Addison (ou insuffisance surrénale primaire chronique), la myasthénie (maladie neuromusculaire qui entraîne une faiblesse musculaire) et l'acromégalie (forme de gigantisme liée à une hypersécrétion de l'hormone de croissance) [1]. Le thymus est alors considéré comme une glande endocrine, au même titre que les autres composantes de ce système comme l'hypophyse, la thyroïde, le pancréas, les surrénales et les gonades.

Avant 1950, la fonction de la glande thymique et de ses lymphocytes demeure inconnue : les souris ayant subi une thymectomie à l'âge adulte ne présentent pas de problème particulier, avec une immunité strictement normale. En revanche, Jacques Miller (Australie, né en 1931) montre qu'une thymectomie réalisée chez des souris juste après leur naissance provoque la mort prématurée des animaux: la présence du thymus à la naissance serait donc essentielle à leur survie. D'autres expériences de Miller montrent que les souris thymectomisées à un jour (et pas plus tard qu'une semaine) sont très sensibles aux infections. Il observe chez ces animaux une lymphopénie dans le sang et les organes lymphoïdes (rate et ganglions lymphatiques). Ces souris sont également incapables de rejeter une greffe de peau étrangère, une réaction immunitaire essentielle. En 1961, il conclut de ses travaux que le thymus est l'organe responsable du développement de lymphocytes immunologiquement compétents [2, 3]. Pour lui, ces lymphocytes constituent une population cellulaire spécifique : les lymphocytes T (T pour thymo-dépendants). Non sans ironie, il écrira plus tard combien ses expériences furent redevables au fait que ses souris avaient été élevées dans un environnement non stérile, favorisant ainsi le développement de leur immunité. $\varepsilon$ n dépit de la justesse des observations de Miller, le rôle du thymus dans le développement des lymphocytes T ne sera néanmoins reconnu que bien des années plus tard. En 1963, le prix Nobel Peter Medawar, écrit encore: «Nous devons considérer la présence de lymphocytes dans le thymus comme un accident de l'évolution sans aucune signification particulière » [4].

C'est toutefois le pédiatre Robert A. Good (1922-2003) (university of Minnesota medical school, Minneapolis, États-Unis) qui revendiqua la découverte du rôle du thymus dans le système immunitaire. Dès 1956 en effet, il décrivait dans la revue Surgery le cas d'un jeune patient associant une tumeur du thymus à un déficit 
acquis en anticorps qu'il essaya de guérir par l'ablation de la tumeur [5]. Good suggère alors l'existence de systèmes lymphocytaires reposant sur deux populations différentes, ces deux systèmes devant cependant être capables de travailler ensemble. En 1961, à Atlantic City, la collaboratrice de Good, Olga Archer, fait sensation à la FASEB (Federation of American Societies of Experimental Biology) en rapportant que l'ablation du thymus chez des lapins à la naissance entraîne un déficit immunitaire avec, en particulier, l'impossibilité de rejeter des greffes. Good accumule des preuves expérimentales et cliniques en faveur du rôle majeur joué par le thymus dans le développement et l'évolution des réponses immunitaires. En 1962, il organise, dans son fief de Minneapolis, le premier congrès sur le thymus où se retrouvent ses concurrents, Jacques Miller, Byron Waksman et Branislav Jankovic (tous deux de Boston), car le thymus est devenu un enjeu majeur de recherche. En 1969, Ivan Roitt (University College London) propose le concept de deux populations, les lymphocytes $T$ (pour thymus) et les lymphocytes B (pour bourse de Fabricius, l'organe responsable chez les oiseaux de la production des anticorps ${ }^{4}$ ) [6]. Chez les mammifères, la moelle osseuse est l'équivalent de la bourse de Fabricius des oiseaux. Avrion Mitchison conclut ensuite à la coopération entre les lymphocytes T (auxiliaires) et les lymphocytes B [7].

À la suite des travaux de Donald Metcalf (1929-2014) sur l'identification des premiers facteurs de croissance, Miller avance l'idée d'un, ou de plusieurs, facteurs thymiques solubles responsables de la différenciation des lymphocytes $T$ [8]. D'innombrables études s'efforcent de caractériser ce(s) facteur(s). Mais il ne sera jamais possible d'identifier avec certitude l'existence réelle d'un tel facteur spécifique du thymus et le modèle endocrine ne pourra être appliqué à la communication entre les cellules épithéliales et les lymphocytes thymiques. La démonstration du rôle essentiel du thymus pendant les vies embryonnaire et fœtale ainsi que l'absence d'effet indésirable de la thymectomie quelques jours après la naissance renforcent l'idée que le thymus est, sinon un organe vestigial, du moins un organe qui devient rapidement inutile, ceci se vérifiant en clinique humaine : le syndrome congénital de Di George, la forme la plus commune de micro-délétion génétique, associe, entre autres anomalies, une absence de thymus à un grave déficit immunitaire; en revanche, les enfants thymectomisés pour corriger une cardiopathie congénitale (une thymectomie étant souvent réalisée pour faciliter le geste du chirurgien) ne présentent pas de déficience immunitaire apparente pendant le reste de leur vie. Cette question mériterait d'être examinée par une étude longitudinale approfondie ${ }^{5}$.

\section{Les interactions entre les systèmes immunitaire et neuroendocrine}

En octobre 1983, la Fondation cardiologique Princesse Liliane organisa à Bruxelles le symposium international Neural Modulation of Immunity

\footnotetext{
${ }^{4}$ D'après des travaux de Bruce Glick sur la bursectomie chez le poulet.

5 Parallèlement au lancement de notre projet intégré FP6 Euro-Thymaide, en 2004, la Commission Européenne a publié une directive interdisant désormais une ablation totale du thymus chez les enfants nécessitant une intervention de chirurgie cardiaque.
}

[9]. Ce symposium était consacré au contrôle exercé par les systèmes nerveux et endocrine sur l'immunité. Il était présidé par le neuroendocrinologue Roger Guillemin, prix Nobel de physiologie ou médecine de 1977, et par deux immunologistes éminents, Melvin Cohn et Theodor Melnechuk. Les intervenants de ce colloque révélaient un tout nouveau domaine de recherche ainsi que différentes approches originales de physiologie intégrée. Au cours de ce symposium, j'appris que les auteurs anglais Ott et Scott avaient rapporté, en 1910, que des extraits d'ovaire et de thymus déclenchaient l'éjection de lait lorsqu'ils étaient injectés à des brebis. Nous savons aujourd'hui que cette action d'éjection du lait est spécifique de l'ocytocine mais cette hormone (la première de nature non stéroïdienne) était alors complètement inconnue puisqu'elle ne sera découverte qu'en 1953 à New York par Vincent du Vigneaud. L'hypothèse d'une synthèse d'ocytocine dans le thymus humain surgit d'emblée et des dosages radio-immunologiques spécifiques révélèrent des quantités importantes d'ocytocine et de neurophysine ${ }^{6}$ dans le thymus humain. Dans cet organe, la coexistence en quantités équivalentes de l'hormone et de sa protéine transporteuse plaidait déjà en faveur d'une synthèse locale. Des extraits de thymus humain furent envoyés à Françoise Acézat, grande spécialiste à l'époque du dosage de l'activité biologique de l'ocytocine, qui observa qu'ils étaient capables de contracter l'utérus de rats femelles. Les quantifications de l'activité biologique de ces extraits étaient également en parfaite concordance avec les dosages radio-immunologiques [10].

Les cellules responsables de la synthèse d'ocytocine dans le thymus sont les cellules épithéliales (CET pour cellules épithéliales thymiques) et non les lymphocytes T qui s'y développent. Parmi les CET, une population particulière, les cellules «nourrices » thymiques, était étudiée depuis plusieurs années par Marie-Paule Defresne, à Liège. Au cours d'une discussion avec elle, survint une hypothèse sous forme de métaphore : les cellules nourrices thymiques nécessiteraient-elles de l'ocytocine pour assister la différenciation des cellules T comme une mère ou une nourrice a besoin d'ocytocine pour allaiter son nourrisson? Cette hypothèse se vérifia à l'expérience et nous démontrâmes que les cellules nourrices thymiques synthétisent bien de l'ocytocine, ce qui n'est pas le cas des lymphocytes T qu'elles enveloppent. Les cellules nourrices thymiques devenaient ainsi un remarquable exemple d'association intime

\footnotetext{
La neurophysine est la protéine (de masse moléculaire apparente de $10 \mathrm{kDa}$ ) de transport de l'ocytocine. Elle est codée par le gène du précurseur de l'ocytocine (le gène codant la pro-oxyphysine).
} 


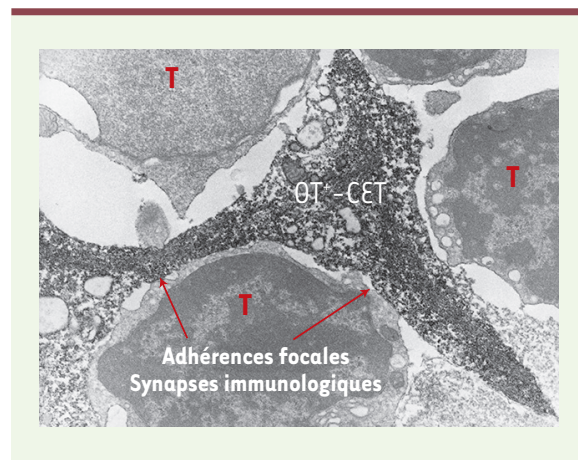

Figure 1. Le microenvironnement thymique. Le prolongement d'une cellule épithéliale thymique de souris positive pour l'ocytocyne peut être détectée (OT $\left.{ }^{+}-C E T\right)$ (coloration en noir par marquage avec un anticorps anti-ocytocine), s'insinuant au milieu de nombreuses cel-

lules $(T)$, thymocytes ou lymphocytes $T$ thymiques. On relève d'abord l'absence complète d'immunoréactivité dans des granules de sécrétion classiquement observée dans les neurones ocytocinergiques [24]. L'image donne l'impression que les thymocytes viennent « téter » la cellule épithéliale et, en ce sens, elle illustre une métaphore entre la lactation contrôlée par l'ocytocine neuro-hypophysaire et le développement des futurs lymphocytes T nécessitant l'ocytocine thymique. En termes scientifiques, ce sont autant de points d'adhérence focale impliqués dans la formation de synapses immunologiques entre CET et lymphocytes T immatures (microscopie électronique réalisée par Martin Wiemann, Münster, Allemagne).

entre deux populations de cellules issues de deux systèmes distincts, le système neuroendocrine et le système immunitaire [11]. En se liant à des récepteurs spécifiques exprimés par les lymphocytes $T$ immatures, l'ocytocine provoque la phosphorylation de kinases liées à l'adhérence focale $[12,13]$ (Figure 1).

En 1986, le premier symposium international de Neuroimmunomodulation eut lieu à Liège sous la présidence de Joseph Wybran et de JeanJacques Vanderhaeghen, respectivement professeurs d'immunologie et de neuropathologie à l'université libre de Bruxelles (ULB) et à partir de 1988, l'étude des interactions entre les systèmes neuroendocrine et immunitaire abordera plusieurs thèmes au sein du réseau d'excellence «Neuroimmunomodulation» (1989-1992) sous l'égide de l'European Science Foundation?

\section{La tolérance immunitaire}

Dès 1900, Paul Ehrlich ${ }^{8}$ (1854-1915) avait proposé la formule « horror autoxicus » (la nature a horreur de l'auto-toxicité) [14] pour affirmer l'impossibilité que l'individu soit agressé en conditions normales par ses propres cellules chargées de sa défense. Ehrlich pensait qu'il devait exister des structures ou des mécanismes pour l'empêcher et que cela était de la plus haute importance pour l'existence de l'individu et la survie des espèces. Dans la foulée de sa théorie révolutionnaire de la sélection clonale, l'immunologiste et virologue Frank Macfarlane Burnet (1899-1985) introduira le terme de tolérance pour caractériser une des propriétés fondamentales du système immunitaire avec sa diversité, sa spécificité et sa mémoire. En 1962, dans une conférence

\footnotetext{
Dont l'auteur a été membre du comité de pilotage.

8 Prix Nobel de physiologie ou médecine de 1908 avec Ilya Ilitch Metchnikov.
}

à l'université de Londres, il déclare: «Si, comme je le pense, le thymus est le site où se déroule la prolifération et la différenciation de lymphocytes en clones dotés de fonctions immunologiques précises, nous devons aussi lui attribuer une autre fonction : l'élimination ou l'inhibition de clones réactifs vis-à-vis du soi » [15]. Burnet qualifiera aussi de clones «interdits » les lymphocytes ayant échappé à la délétion clonale.

En 1976, Susumu Tonegawa9 explique l'extrême diversité de la réponse immunitaire vis-à-vis du non-soi par l'identification d'un mécanisme moléculaire de recombinaison aléatoire au niveau des segments de gènes codant les domaines variables du récepteur de l'antigène des lymphocytes B (le BCR) [16]. En 1984, Tak Mak et Mark Davis montrent qu'un mécanisme analogue existe dans le thymus pour la diversification du récepteur de l'antigène des lymphocytes T (le TCR) [17-19] qui reconnaît le complexe formé par l'antigène avec une protéine $d u C M H$. Le TCR présente une homologie proche des immunoglobulines, plus lointaine avec le CMH. La parenté de ces molécules renforce l'homogénéité du système immunitaire et suggère des mécanismes de réplication et de diversification tout au long de l'évolution.

Plusieurs millions de combinaisons résultent de la fantastique loterie à l'œuvre dans la génération de la diversité immunitaire (generation of diversity ou GOD pour les anglo-saxons) et une grande proportion de ces combinaisons est capable de reconnaître les structures moléculaires de l'hôte (le soi). Le système immunitaire n'agresse cependant pas, dans les conditions normales, le soi et, pendant longtemps, l'immunologie a été définie comme la science de la discrimination du soi et du non-soi, évoquant une question presque d'ordre philosophique. Frank Macfarlane Burnet parlait bien de l'immunologie comme de la «science du soi et du non-soi ». Pourtant les lymphocytes sont incapables d'effectuer cette distinction entre soi et non-soi. En 1987 et 1988, les équipes de Nicole Le Douarin (Institut d'embryologie cellulaire et moléculaire du CNRS et du Collège de France) [20], John Kappler et Philippa Marrack (à Denver, États-Unis) [21], Hugh Robson Macdonald (à Epalinges, Suisse) [22] et Harald von Boehmer (à Bâle, Suisse) [23] démontrent expérimentalement la théorie de la sélection clonale initialement envisagée par Burnet : les lymphocytes doivent d'abord être éduqués dans le thymus à reconnaître et à tolérer les antigènes du soi. L'équipe de von Boehmer en a donné en effet une démonstration élégante, en 1988, en générant

\footnotetext{
9 Prix Nobel de physiologie ou médecine en 1987 pour sa découverte du principe
} génétique de la génération de la diversité des anticorps. 
des souris transgéniques dont les lymphocytes $T$ exprimaient un seul type de TCR spécifique d'un antigène présent seulement chez les souris mâles. Le thymus de ces souris mâles était pauvre en lymphocytes contrairement au thymus des souris femelles qui, lui, regorgeait de thymocytes. Ceci ne pouvait s'expliquer que par l'élimination des lymphocytes $T$ transgéniques due à la présence de l'antigène masculin dans le thymus des souris mâles, une situation impossible dans le thymus des souris femelles. Ainsi, le thymus apparaissait, d'abord, comme un cimetière pour lymphocytes $T$ : sur cent précurseurs de lymphocytes T qui migrent de la moelle osseuse vers le thymus, seuls deux à cinq en ressortent dans un état de tolérance vis-à-vis du soi, et de compétence contre le non-soi.

À la même époque, la communauté scientifique était confrontée à un problème fondamental de biologie cellulaire. L'ocytocine avait permis d'établir le modèle classique de la sécrétion d'une hormone par des neurones (la neurosécrétion). Cependant, les CદT en culture, même stimulées de diverses manières, ne sécrétaient absolument pas la moindre trace d'ocytocine, ce qui était en opposition non seulement avec la vérité scientifique établie pour l'ocytocine, mais aussi avec les travaux montrant l'existence d'une production d'ocytocine dans les CET, et l'expression de récepteurs spécifiques de l'hormone par les lymphocytes thymiques. Pourtant, Martin Wiemann avait publié un article de microscopie électronique qui confirmait la présence d'ocytocine dans le cytosol des lymphocytes thymiques mais pas dans les granules sécrétoires issus de l'appareil de Golgi (Figure 1) [24].

Un problème se posait donc: si l'ocytocine n'est pas sécrétée, ou au moins libérée, par les CET, comment peut-elle se lier à des récepteurs spécifiques qui sont exprimés par les lymphocytes T thymiques? S'agissait-il d'une expression « illégitime » du gène de l'ocytocine dans l'épithélium thymique ? Toutes les hypothèses, y compris celle d'un nouveau membre de la famille des peptides neuro-hypophysaires, ont été expérimentées puis écartées. Plusieurs observations permirent peu après de comprendre la signification de la synthèse d'ocytocine dans le thymus. À la fin de l'année 1990, I'australien John Funder, grand expert mondial de la physiologie des glandes surrénales, proposa un nouveau modèle de communication cellulaire, la communication cryptocrine (du grec cryptos, caché), mot signifiant l'absence d'une sécrétion détectable de l'hormone. Selon Funder, qui avait auparavant travaillé avec Frank Macfarlane Burnet sur l'existence de récepteurs des hormones surrénaliennes dans le compartiment épithélial du thymus, deux exemples de communication cryptocrine existent dans le corps : dans le testicule d'une part, entre les grandes cellules de Sertoli et les spermatozoïdes dont elles contrôlent la différenciation, et dans le thymus d'autre part, entre les cellules épithéliales/nourrices et les petits lymphocytes T dont elles régulent le développement [25]. À cette même époque furent déchiffrés par le laboratoire de HansGeorg Rammensee (institut Max-Planck, Tübingen, Allemagne) les mécanismes biochimiques responsables de la présentation des antigènes par les protéines du CMH exprimées à la surface de différentes cellules présentatrices d'antigène [26]. L'hypothèse selon laquelle l'ocytocine se comporterait dans le thymus non pas comme la neurohormone sécrétée mais plutôt comme un antigène du soi, appartenant à la famille des hormones neuro-hypophysaires, qui serait présenté aux lymphocytes T pendant leur éducation à la tolérance centrale au sein de cet organe fut formulée. Les expériences montrèrent que cette hypothèse correspondait bien à la réalité. La présentation de l'ocytocine thymique se produit à la surface des CET. Elle n'implique aucune sécrétion. Elle est en fait responsable de la programmation de la tolérance du système immunitaire vis-à-vis des fonctions neurohypophysaires assurées par l'ocytocine et la vasopressine. Contrairement à la vasopressine, une hormone antidiurétique, l'ocytocine est fortement exprimée dans le thymus. La tolérance immunitaire vis-à-vis de l'ocytocine est donc beaucoup plus importante que celle développée vis-à-vis de la vasopressine. Ceci expliquait pourquoi les lapins réagissaient si faiblement aux immunisations avec l'ocytocine réalisées antérieurement dans notre laboratoire. La tolérance immunitaire vis-à-vis de l'ocytocine était si forte qu'elle était extrêmement difficile à briser, contrairement à celle de la vasopressine.

Sur la base de ces travaux, nous avons proposé un modèle qui transpose à l'échelle moléculaire le double rôle du thymus dans la différenciation des lymphocytes T, à la fois dans le développement des lymphocytes T (Ia thymopoïèse) et dans la programmation de la tolérance immunitaire centrale. Ce modèle repose sur les deux types de comportement que l'ocytocine peut adopter dans le thymus (Figure 2). En tant que signal cryptocrine ciblé à la surface des CET, l'ocytocine peut se lier à un récepteur neuroendocrine spécifique exprimé par les lymphocytes T entrant en contact des cellules épithéliales. Cette liaison provoque la mobilisation de seconds messagers (inositol triphosphate, InsP3 ou IP3) à l'intérieur des thymocytes ainsi que l'activation de protéines de l'adhérence focale. L'ocytocine thymique serait ainsi capable de stimuler la formation des «synapses » entre CहT et thymocytes [27] qui jouent un rôle majeur dans le contrôle exercé par l'épithélium thymique sur la différenciation des lymphocytes T. Dans le même temps, l'ocytocine thymique se comporte aussi comme un antigène du soi de la famille neuro-hypophysaire. Elle est alors présentée par les protéines du CMH exprimées par les CET, cette présentation permettant l'élimination (ou la sélection négative) des lymphocytes T qui expriment un récepteur TCR spécifique de la combinaison CMH/ ocytocine. Cette présentation suivie de cette liaison au TCR serait responsable de l'établissement de la tolérance immunitaire centrale vis-à-vis des fonctions neuro-hypophysaires. Ce modèle a été ensuite appliqué avec succès aux autres membres de différentes familles neuroendocrines exprimés par les CET comme la 


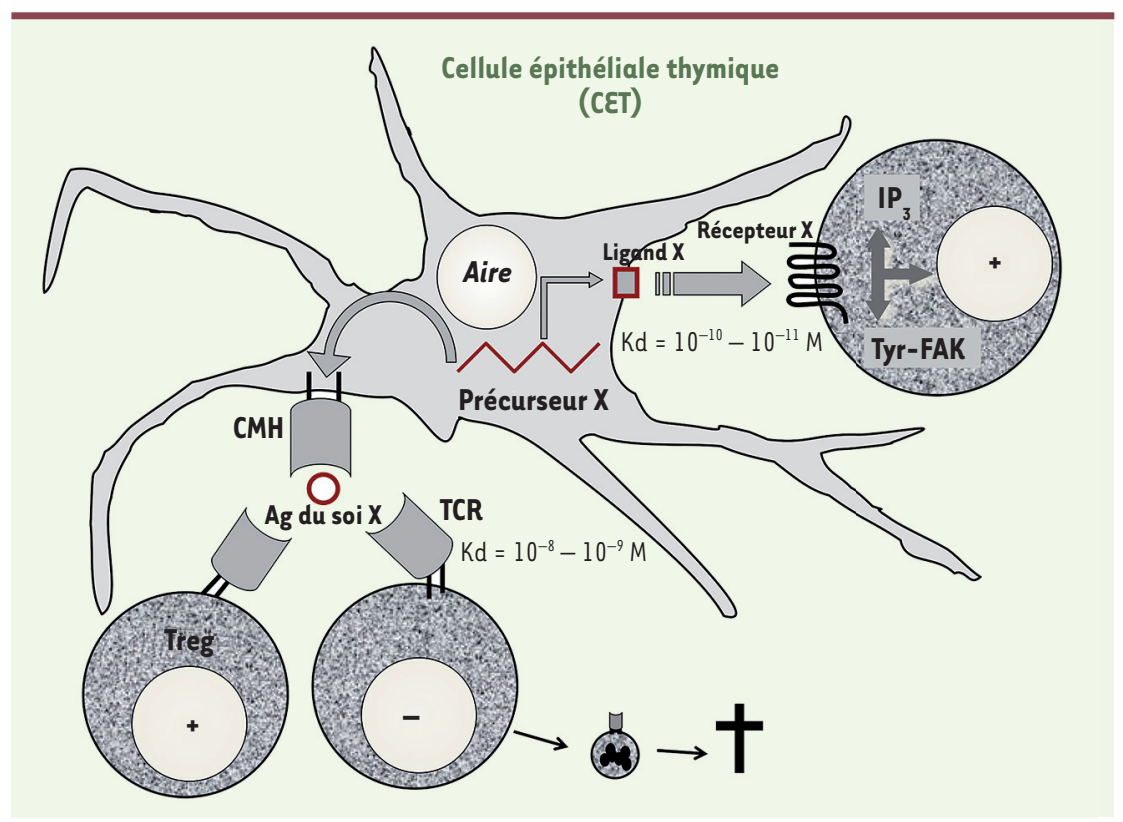

Figure 2. Le double rôle des précurseurs neuroendocrines dans la différenciation des lymphocytes $T$. Ce modèle théorique récapitule à l'échelle moléculaire le double rôle du thymus dans le développement des lymphocytes $T$ et la programmation de leur tolérance aux principes neuroendocrines. À la suite à sa transcription sous le contrôle de Aire dans le noyau des cellules épithéliales thymiques CET, un précurseur neuroendocrine « $X$ » peut être apprêté pour donner deux types de signaux bien distincts. D'une part, il peut être la source d'un ligand cryptocrine $X$ capable de se lier à des récepteurs spécifiques exprimés par les thymocytes et de mobiliser des seconds messagers (comme l'inositol triphosphate, IP3) et de phosphoryler des protéines (comme p125FAK [focal adhesion kinase] dans le cas de l'ocytocine thymique). Cette signalisation intervient donc comme un signal accessoire positif dans le développement des lymphocytes T. D'autre part, le même précurseur neuroendocrine $X$ peut donner naissance à un antigène $(\mathrm{Ag})$ du soi $X$ présenté par les protéines du complexe majeur d'histocompatibilité (CMH) exprimées par les CET. Pendant la vie fœtale, cette présentation aboutit à la sélection négative des lymphocytes T exprimant aléatoirement un TCR ( $T$ cell receptor) spécifique de ce complexe $\mathrm{CMH} / \mathrm{Ag}$ du soi $\mathrm{X}$. Après la naissance, cette même présentation aboutit à la génération de lymphocytes $T$ régulateurs (Treg) spécifiques du même complexe.

neurokinine A, pour la famille des tachykinines, le neuropeptide y [28] et le facteur de croissance apparenté à l'insuline de type 2 (IGF[insulin growth factor]-2), pour la famille de l'insuline [29, 30].

Le thymus programme donc la tolérance immunitaire au soi neuroendocrine via la transcription, dans les CET, de gènes apparentés. Un principe économique réside dans l'organisation du répertoire intra-thymique de ces gènes neuroendocrines : un membre dominant par famille y est en effet exprimé. Toutefois, les précurseurs neuroendocrines thymiques ne sont pas apprêtés selon le modèle de la neurosécrétion classique. Ils sont en fait apprêtés comme la source d'antigènes du soi qui sont ensuite présentés par les protéines thymiques du CMH [31]. Ce nouveau paradigme d'antigènes du soi neuroendocrine, très spécifique du thymus, a permis une coévolution intégrée et harmonieuse des systèmes neuroendocrine et immunitaire lorsque les gènes activateurs de la recombinaison des BCR et TCR, et la réponse immunitaire adaptative subséquente, ont émergé chez les poissons cartilagineux il y a environ 450 millions d'années [32-34] (voir plus loin).

Les travaux de Bruno Kyewski et ses collaborateurs ont ensuite montré que les CET, surtout d'origine médullaire, étaient le site de l'expression de nombreux antigènes représentatifs de tissus périphériques [35]. La publication dans Annual Review of Immunology a définitivement assis le rôle central du thymus dans la tolérance immunitaire centrale au soi [36]. Contrairement aux antigènes du soi neuroendocrine, la plupart des antigènes spécifiques de tissu n'exercent pas de signalisation accessoire pendant le développement intrathymique des lymphocytes $T$.

En 1972 Richard Gershon, à Yale, avait mis en évidence des cellules immunitaires suppressives assurant une régulation des cellules immu- nitaires [37]. II parlait avec humour de la suppression comme «la deuxième loi de la thymodynamique ». Après sa mort prématurée, ses travaux ont été repris, en 1995, par le japonais Shimon Sakagushi qui découvre un autre mécanisme tolérogène majeur dépendant du thymus [38]. Après la naissance, le thymus est en effet la source d'une population de cellules $T$ régulatrices capables d'inhiber en périphérie les lymphocytes $T$ réactifs au soi ayant échappé au filtre de la sélection négative intra-thymique. La génération de ces lymphocytes T régulateurs dépend aussi de la présentation d'antigènes du soi par les protéines thymiques du CMH.

\section{Le thymus et la faillite de la tolérance : l'auto-immunité}

L'avènement de la tolérance immunitaire finira par aboutir à la redéfinition de ce qui est antigénique, c'est-à-dire capable de stimuler une réponse immunitaire (réponse immunogène), et de ce qui ne l'est pas (réponse tolérogène). Après l'anémie hémolytique auto-immune décrite par William Dameshek, hématologue à Boston, c'est la thyroïdite de Hashimoto qui devient un autre modèle de maladie auto-immune à la suite des travaux de Ernst Witebsky ${ }^{10}$. Avec son jeune

\footnotetext{
10 Présentés au $1^{\text {e }}$ congrès d'Immunopathologie à Bâle en 1956.
} 
élève Noël Rose, Witebsky identifie les premiers auto-anticorps antithyroïdiens, ce que confirment peu de temps après, à Londres, Deborah Doniach et Ivan Roitt. Néanmoins, Witebsky n'excluait pas du tout l'idée que les anticorps anti-thyroïdiens ne soient que des marqueurs et non des effecteurs du processus pathogène. En 1965, Witebsky et Dameshek organisent à New York le premier colloque officiel sur l'auto-immunité. À partir de ce moment, le nombre des maladies candidates à une origine auto-immune ne va cesser d'augmenter. Les principales sont le diabète juvénile insulino-dépendant (le diabète de type 1), la plupart des maladies de la thyroïde, la maladie d'Addison (une insuffisance surrénalienne), la polyarthrite rhumatoïde, la sclérose en plaques et le psoriasis. Comme l'écrira Anne-Marie Moulin [39], médecin et philosophe des sciences au CNRS, l'auto-immunité accomplit ainsi une nouvelle révolution :

Elle est une exception à la fondamentale exclusion du soi du domaine de l'immunisation, une disparition de la différence constitutive entre soi et non-soi ;

Elle peut être perçue comme un élément dynamique dans les relations fluctuantes que le soi contracte avec le non-soi.

La mise en évidence d'un rôle essentiel du thymus dans la programmation centrale des fonctions neuroendocrines soulevait intuitivement la question de savoir si un dysfonctionnement du thymus pouvait jouer un rôle dans la pathogénie d'une endocrinopathie auto-immune majeure comme le diabète de type 1 . Pour résoudre cette question, l'expression des gènes de la famille de l'insuline a été étudiée ${ }^{11}$ dans le thymus de rats BioBreeding (BB), qui est, avec la souris NOD (non obese diabetic), un modèle animal classique du diabète de type 1 humain. Alors que les gènes de l'insuline et de l'IGF-l sont normalement exprimés dans le thymus de tous les rats BB examinés, l'expression de l'IGF-2 est déficiente dans la très grande majorité des thymus des rats $B B$ susceptibles au diabète auto-immun. Cette déficience de l'expression de I'IGF-2 dans le thymus des rats BB permettrait d'expliquer la chute du nombre de lymphocytes $T$ (lymphopénie) caractéristique de ces animaux, lymphopénie qui touche aussi les cellules T régulatrices [40]. L'identification du gène Aire (auto-immune regulator) joua un rôle majeur dans l'adhésion définitive de la communauté scientifique à l'idée qu'un défaut de la tolérance thymique joue un rôle essentiel dans la physiopathologie des maladies autoimmunes [50] $(\rightarrow)$. En 1997, un consortium germano-finlandais identifia le gène responsable d'un très rare syndrome congénital chez les nouveau-nés, caractérisé par une auto- $(\rightarrow)$ Voir la Synthèse de N. Lopes et al., $m / s n^{\circ} 8-9$, août-septembre 2015, page 742 immunité multiple associant une insuffisance des parathyroïdes (avec hypocalcémie), une insuffisance des surrénales (maladie d'Addison) et des épisodes récurrents d'infections des muqueuses par la levure Candida albicans. Les travaux réalisés dans le laboratoire de Diane Mathis ont montré, en 2002, que l'expression de Aire était maximale dans l'épithélium du thymus, et que les souris transgéniques présentant une invalidation de ce gène développaient plusieurs manifestations auto-immunes. Cette équipe montra également que l'expression

${ }^{11}$ En collaboration avec Dale Greiner (université du Massachussets, Worcester, États-Unis). de nombreux gènes était diminuée dans les CET des souris transgéniques, notamment ceux codant l'ocytocine, I'IGF-2, l'insuline et le neuropeptide $y$, identifiés auparavant. Les auteurs concluaient leur article en disant que Aire contrôle l'expression de nombreux gènes dans le thymus et qu'en son absence, la diminution de l'expression intra-thymique des autres gènes permet le développement de réactions auto-immunes dirigées contre les antigènes tissulaires périphériques qu'ils codent [41]. II devint ainsi évident que le gène Aire exprimé de manière prédominante dans l'épithélium thymique était un rempart majeur contre l'auto-immunité. Le nombre de publications sur Aire s'envola ensuite et le retour à la tolérance centrale sous la dépendance du thymus devint le sujet principal des laboratoires intéressés dans la physiopathologie des maladies autoimmunes spécifiques d'organes. Plus récemment, il a été montré qu'un deuxième gène, Fezf2 (fasciculation and elongation protein zeta [FEZ] family zinc finger 2), contrôle la transcription intra-thymique d'antigènes du soi indépendants de Aire $[42,51](\rightarrow)$.

Les fonctions et la tolérance immunitaires vis-à-vis des membres de la famille de l'insuline ont été aussi étudiées en utilisant des souris transgéniques présentant une délétion du gène codant I'IGF-2. Les souris $\operatorname{lgf} 2^{-/-}$ présentent des altérations dans la différenciation d'une population de cellules présentatrices d'antigènes, les cellules dendritiques. Leur tolérance vis-à-vis de l'IGF-2 est perdue (comme cela était attendu puisque I'IGF-2 n'est plus un antigène du soi chez ces souris mutées), mais surtout, bien qu'elles ne développent pas de diabète auto-immun, leur tolérance vis-à-vis de l'insuline est beaucoup plus faible que celle de souris sauvages [43]. L'expression de I'IGF-2 semble donc nécessaire pour l'installation d'une tolérance complète vis-à-vis de l'insuline, et l'homologie importante entre l'IGF-2 et l'insuline est à l'origine d'une tolérance croisée entre ces deux membres de la même famille de protéines. Dans des expériences encore préliminaires, ces observations ont été vérifiées chez des souris présentant une délétion de l'IGF-2 spécifiquement dans les CET.

L'absence (ou la rupture) de la tolérance immunitaire à l'égard de la famille de l'insuline est une condition nécessaire, mais non suffisante, pour l'apparition d'un diabète de type 1. Pourtant, chez les jumeaux homozygotes, la concordance de ce type de diabète n'est que de 30 à $40 \%$. Cette discordance souligne l'importance des facteurs génétiques prédisposant à cette maladie mais également l'intervention de facteurs environnementaux comme des infections, en particulier par les entérovirus. 
Physiologie du thymus

- Expression contrôlée par Aire et Fezf2 de gènes neuroendocrines ou codant des antigènes tissulaires

- Sélection négative de lymphocytes T spécifiques de ces antigènes

- Génération de lymphocytes T régulateurs spécifiques de ces antigènes

Physiopathologie du thymus

- Absence/diminution de l'expression de gènes neuroendocrines ou codant des antigènes tissulaires

- Enrichissement du répertoire T périphérique avec des clones T « interdits » réactifs au soi (Teff)

- Diminution de la génération de lymphocytes T régulateurs (Treg) spécifiques du soi

Une condition nécessaire mais non suffisante pour déclencher une maladie auto-immune

Pont entre lymphocytes $T$ « interdits » et auto-antigènes périphériques

- Rôle de facteurs environnementaux

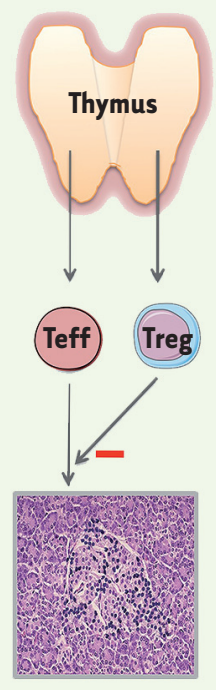

Figure 3. Le rôle central du thymus dans la programmation de la tolérance immunitaire au soi et le développement de l'auto-immunité spécifique d'organes. Dans les conditions normales, sous le contrôle des gènes Aire et Fezf2, l'épithélium thymique exprime des gènes apparentés aux principales familles neuroendocrines et des gènes codant de nombreux antigènes tissulaires. La présentation par le complexe majeur d’histocompatibilité (CMH) thymique des antigènes du soi neuroendocrine conduit à la fois à l'élimination des lymphocytes T spécifiques de ces antigènes (sélection négative) ainsi qu’à la génération de lymphocytes T régulateurs spécifiques de ces antigènes. Ce double mécanisme est responsable de la programmation de la tolérance immunitaire centrale vis-à-vis des fonctions neuroendocrines. Dans certaines conditions pathologiques, la diminution de l'expression et de la présentation intra-thymiques des antigènes du soi neuroendocrine aboutit à la libération progressive dans le sang de clones T effecteurs « interdits » (Teff) dirigés contre ces antigènes en même temps qu'à la diminution de la production de lymphocytes $T$ régulateurs (Treg) spécifiques de ces antigènes. Ces deux phénomènes sont nécessaires mais non suffisants pour le développement d'une maladie auto-immune. Pour qu'une maladie auto-immune apparaisse, des facteurs environnementaux encore mal identifiés interviennent aussi. Ces facteurs environnementaux comprennent des virus, la nature de l'alimentation, le stress et une déficience en vitamine D.

L'hypothèse qu'une infection par le virus Coxsackie B4 (CV-B4) pourrait induire un dysfonctionnement du thymus et une perturbation de la tolérance centrale vis-àvis de la famille de l'insuline est explorée depuis plus de quinze ans ${ }^{12}$. Le virus CV-B4 est capable d'infecter les compartiments épithélial et lymphoïde des thymus de I'homme et de la souris. II est à l'origine d'une diminution importante des thymocytes et d'une surexpression des molécules du CMH à la fois par les CET et par les lymphocytes $\mathrm{T} C D 4^{+} \mathrm{CD} 8^{+}$immatures. L'infection par le virus CV-B4 de cultures de cellules thymiques isolées de fœtus de souris a également un important impact sur la différenciation des lymphocytes T. L'infection d'une lignée de CET médullaires murines provoque également une diminution drastique de l'expression de l'IGF-2 dans ces cellules alors que les expressions de l'IGF-l et de l'insuline ne sont pas affectées. L'infection du thymus par le virus CV-B4 est donc capable de perturber la tolérance thymique centrale vis-à-vis des protéines de

12 Collaboration avec l'équipe de Didier Hober (service de virologie, pôle de biologie pathologie génétique, CHRU de Lille). la famille de l'insuline et d'augmenter la virulence du virus en promouvant la tolérance centrale à son égard $[44,45]$.

$\varepsilon n$ ce qui concerne les thyroïdites auto-immunes qui constituent les maladies endocrines auto-immunes les plus fréquentes, tous les antigènes thyroïdiens (thyroperoxydase, thyroglobuline et récepteur de la TSH [thyroid-stimulating hormone] ou thyrotrophine) sont exprimés dans les cellules épithéliales thymiques en conditions normales. Comme cela avait été décrit pour la première fois par J.A. Hammar, un accroissement important de la taille du thymus (hyperplasie) est souvent observé dans l'hyperthyroïdie de Graves-Basedow ; cette hyperplasie disparaît progressivement avec le traitement de la maladie $[46,47]$.

L'IGF-2 est donc le précurseur thymique tolérogène dominant de la famille de l'insuline. Un dysfonctionnement thymique joue un rôle moteur dans le développement de la réponse auto-immune diabétogène et l'infection du thymus par le virus CV-B4 pourrait être impliquée dans la pathogénie du diabète de type 1 . Le très faible niveau d'expression dans les CET de la protéine qui représente l'autoantigène primaire ciblé dans le diabète de type 1 , serait à l'origine de son importante immunogénicité. Fondé sur les propriétés tolérogènes de I'IGF-2, un «self-vaccin négatif » contre le diabète de type 1 est en cours de développement [48]. L'existence d'une programmation intra-thymique de la tolérance immunitaire vis-à-vis des protéines 


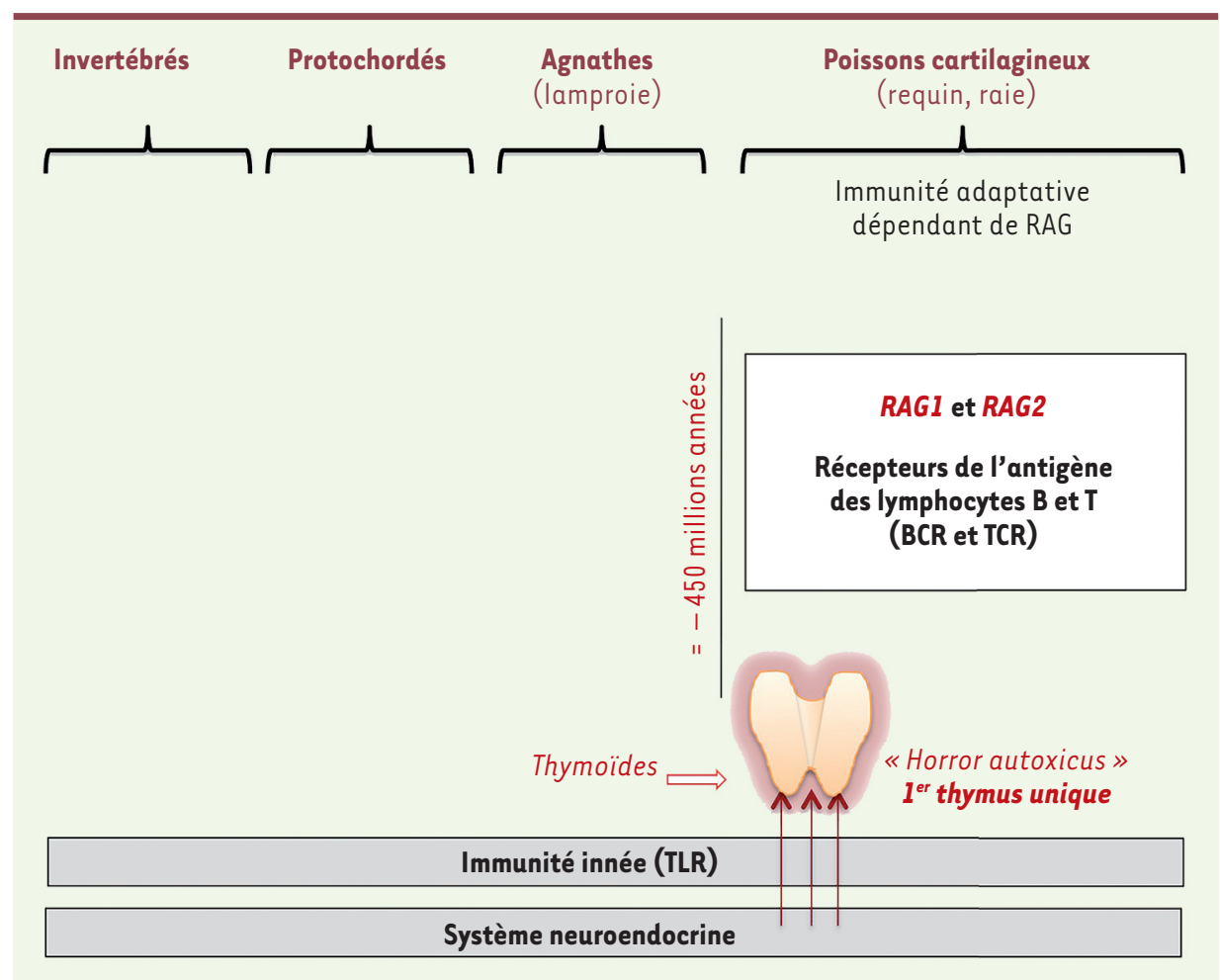

Figure 4. Évolution intégrée des systèmes neuroendocrine et immunitaire. Depuis toujours et encore aujourd'hui, le système neuroendocrine et l'immunité innée évoluent en parallèle et en parfaite harmonie. Les récepteurs Toll-like (TLR) ne peuvent entrer en effet en jeu contre le soi normal et non endommagé. Le premier thymus unique est apparu chez les poisons cartilagineux avec l'émergence de l'immunité adaptative. Cette dernière dépend de l'apparition à cette époque de deux enzymes, les gènes activateurs de recombinases $R A G$ (recombination-activating gene) 1 et $R A G 2$, capables de générer l'extrême diversité des BCR (B cell receptor) et TCR (T cell receptor). La présentation des antigènes du soi neuroendocrine dans le thymus peut être considérée a posteriori comme le moyen le plus efficace et le plus économique pour l'éducation du système immunitaire adaptatif à reconnaître et à tolérer le soi neuroendocrine dès les premières étapes de sa différenciation.

des familles neuroendocrines était une nécessité absolue pour la préservation de l'homéostasie générale. Comme de nombreux travaux l'ont montré, la plupart des hormones et des neuropeptides exercent un contrôle sur les réponses immunitaire et inflammatoire. Ils se lient à des récepteurs neuroendocrines qui sont exprimés par les cellules immunocompétentes et les activent. Si une tolérance immunitaire à ces signaux et à ces récepteurs neuroendocrines n'était pas fermement établie, le risque de développer des réactions auto-immunes à l'égard de ces molécules serait très élevé et compromettrait la survie des espèces.

La Figure 3 résume le modèle actuel décrivant le rôle de l'épithélium thymique comme «bouclier » contre l'auto-immunité et d'un dysfonctionnement du thymus comme un « moteur » des maladies autoimmunes spécifiques d'organes.

\section{Conclusion. Le rôle du thymus au cours de l'évolution (Figure 4)}

Le rôle du thymus est considérable en immunologie et il peut être reconsidéré du point de vue de l'évolution. Au temps où a émergé l'immunité adaptative dépendant des gènes activateurs de la recombinase RAG (recombination-activating gene) l et RAG2, le thymus serait apparu il y a environ 450 millions d'années chez les poissons cartilagineux. Des formations thymoïdes dispersées dans l'appareil branchial des lamproies ont précédé l'avènement du thymus unique. Elles exprimaient déjà Foxn4 (forkhead box N4), le paralogue de Foxn 1, le facteur de transcription spécifique de la différenciation de l'épithélium thymique [49]. L'apparition du thymus a permis l'orchestration de la tolérance centrale vis-à-vis du soi, rempart nécessaire contre le risque élevé d'auto-toxicité inhérent à la loterie du nouveau système immunitaire adaptatif. Une défaillance génétique (mutations de Aire ou Fezf2) ou acquise (comme l'infection persistante des (ET par le virus (V-B4) de la fonction tolérogène thymique joue un rôle nécessaire, mais non suffisant, dans l'apparition des maladies auto-immunes spécifiques d'organes qui seraient in fine le tribut payé, par l'espèce humaine surtout, en échange d'une immunité adaptative efficace et de plus en plus complexe. $\diamond$

\section{SUMMARY}

History of the thymus: from an "accident of evolution" to the programming of immunological self-tolerance

This synthesis presents the most important disruptions of conceptions about the thymus since its discovery in Antique Greece. For centuries, the thymus was considered as a vestigial organ, and its role in T-lymphocyte differentiation has been proposed only in the 1960's. Most recent studies attribute to the thymus an essential and unique role in the programming of central immunological 


\section{Thymus... du grec, excroissance}

\section{Armelle Debru}

Le grec dispose de deux mots différents par leur accentuation : $\Theta u ́ \mu o \zeta$ et $\theta u \mu o ́ s$. Le premier, accentué sur le $v$, masculin ou neutre, est anciennement un nom de plante (sarriette). Le second terme a été très utilisé en philosophie pour désigner la passion, l'ardeur. Ils ne doivent pas être confondus.

Comme terme médical, thumos apparaît avec le sens d'« excroissance », dans le traité pseudo-hippocratique De alimento 17 : « excrétions... par hémorroïde, excroissance (thumon), dartre, tumeur, chancre... ». Sens qui se retrouve dans Galien, De tumoribus praeter naturam («Sur les gonflements contre nature ») VII, 731. Sous le terme générique de onkos, gonflement, Galien rassemble diverses sortes d'altérations, comme le phlegmon, le chancre, le polype, etc. Parmi ceux « qui n'ont pas une enflure remarquable » il classe les thymus (thumoi) et « autres excroissances charnues de ce type ».

Cependant, au-delà de ce sens générique, Galien désigne aussi plus spécifiquement par thumos un corps glanduleux qui se situe à l'arrière du sternum. II s'agit bien pour lui d'une glande. Cette notion est déjà présente dans le Corpus Hippocraticum (Ve siècle avant JC), notamment dans le traité «Sur les glandes (peri adenôn) ». Elles y sont présentées comme étant « de nature spongieuse, lâches et grasses ». Cependant aucune n'est appelée thymus. Chez Galien, qui l'a désormais bien identifiée (sans doute toutefois à la suite d'autres anatomistes qui n'ont pas transcrit leurs observations), sa fonction est essentiellement protectrice : celle de préserver la sécurité de la veine cave.

Sa description la plus détaillée est un long passage du traité sur L'Utilité des parties, livre VI, chap. 4 : « De chaque côté de la poitrine sont les deux grands lobes du poumon; quant au cinquième et petit lobe, placé du côté droit, dans l'intérêt de la veine cave, il s'étend depuis le diaphragme jusqu'à l'oreillette du cœur. À cet endroit une des parties de la veine cave s'insère sur le cœur ; l'autre, la plus volumineuse, s'élève directement vers la fourchette; elle est déviée jusqu'à un certain point par les prolongements du cœur et s'appuie ensuite sur le corps appelé thymus. Cette glande, si grosse et si molle à la fois, la nature l'a étendue à la partie supérieure de la face interne de l'os médian du thorax appelé sternum, de façon à ce que cet os ne touche pas la veine cave... Partout, en effet, où la nature divise un vaisseau suspendu, elle établit toujours une glande au milieu même de la séparation pour combler l'intervalle ... [La fin du passage offre des difficultés de lecture des manuscrits] : «La nature a fourni un très grand secours à toutes les ramifications des veines, et avant tout à la veine cave ellemême, en établissant proche des os, comme une séparation tout à fait semblable à un tissu foulé cette glande dont nous venons de parler et qui devait en même temps fournir une base de sustentation et donner à toutes les parties de la veine une grande sécurité » (traduction : Daremberg, Fuvres anatomiques, physiologiques et médicales de Galien, Paris, Baillière, 1856, I p. 391-393).

self-tolerance. The basal mechanism implicated in this function is the transcription in thymic epithelium of genes encoding precursors of self-antigens. Processing of these latter leads to presentation of self-antigens by the major histocompatibility complex (MHC) machinery expressed by thymic epithelial cells and dendritic cells. During fetal life, this presentation drives negative selection of T-cell clones harboring receptors with high affinity for these $\mathrm{MHC} /$ self-antigen complexes. After birth, this presentation also promotes the generation of regulatory $T$ cells specific for these complexes. A number of studies, as well as the identification of Aire and Fezf2 genes, have shown that a thymus dysfunction plays a crucial role in the development of organ-specific autoimmunity. $\diamond$

\section{REMERCIEMENTS}

Je souhaite exprimer ici ma plus vive gratitude au $\operatorname{Pr}$ Anne Marie Moulin dont le livre « Le dernier langage de la médecine - Histoire de l'immunologie de Pasteur au SIDA » m'a considérablement aidé dans ma transition de l'endocrinologie à l'immunologie. Elle a de plus porté une attention minutieuse à l'édition de cet article.
Je remercie tout particulièrement le Pr Armelle Debru pour son éclairage d'expert à propos de l'apparition du mot "thymus" dans l'œuvre de Galien (voir Encadré). Nos travaux ont été soutenus par le Fonds Léon Fredericq du CHU de Liège, les Fonds Spéciaux à la Recherche de l'Université de Liège, la Wallonie, le F.R.S.-FNRS de Belgique, la Fédération Wallonie-Bruxelles, le Fonds Alphonse Rahier pour la recherche en diabétologie (Belgique), la Commission européenne (Projet Intégré FP6 Euro-Thymaide 2004-2008), l'European Association for Study of Diabetes (EASD, Germany), et la Juvenile Diabetes Research Foundation (JDRF, New York). Je souhaite remercier du fond du cœur mon équipe du GIGA-I Centre d'Immunoendocrinologie à l'Université de Liège qui a tant contribué à la cohérence de ces travaux au long de ces trente dernières années : les Drs Henri Martens, Denis Franchimont, Sophie Perrier d'Hauterive, Fabienne Brilot, Isabelle Hansenne, Olivier Dardenne, Christel Pequeux, Grégory Rasier, Céline Gerard, Ouafae Kecha-Kamoun, Imane Achour, Virginie Gridelet, Yves Vanneste, Françoise Robert (INSERM), Béatrice Goxe et Hamid Kermani, MMes Chantal Renard, Marie-Thérèse Hazée-Hagelstein, Francine Louis, Yvonne Vrindts-Gevaert ${ }^{+}$, Barbara Polese, Gwennaëlle Bodart, Hélène Michaux et Nadine Cormann-Goffin, MM. Eric Vandersmissen, Khalil Farhat, Aymen Halouani et Abdellah Benhida. Je remercie aussi pour leur précieuse collaboration de très longue date le Pr Didier Hober (Université de Lille II) et le Dr Hela Jaïdane (Faculté des Sciences El Manar de Tunis et Université de Monastir, Tunisie). 


\section{LIENS D'INTÉRÊT}

L'auteur déclare n'avoir aucun lien d'intérêt concernant les données publiées dans cet article.

\section{RÉFÉRENCES}

1. Hammar JA. The new views at the morphology of the thymus gland and their bearing on the problem of the function of the thymus. Endocrinology $1921 ; 5: 543-73$.

2. Miller JF. Immunological function of the thymus. The Lancet 1961 ; II : 748-9.

3. Miller JF. The thymus and the development of immunologic responsiveness. Science $1964 ; 144$ : 1544-51

4. Medawar PB. Discussion after Miller JFAP and Osoba D. Role of the thymus in the origin of immunological competence. In: Wolstenholme GEW, Knight J (eds) The Immunologically Competent Cell: Its Nature and Origin (vol. 16), London : Ciba Foundation Study Group, 1963.

5. Greaves MF, Roitt IM, Rose ME. Effect of bursectomy and thymectomy on the responses of chicken peripheral blood lymphocytes to phytohaemagglutinin. Nature $1969 ; 220: 293-5$.

6. Good RA, MacLean LD, Varco RL, Zak SJ. Thymic tumor and acquired agammaglobulinemia : a clinical and experimental study of the immune response. Surgery $1956 ; 40: 1010-7$.

7. Mitchison NA. The carrier effect in the secondary response to hapten-protein conjugates. II. Cellular cooperation. Eur J Immunol 1971 ; 1 : 18-27.

8. Osoba D, Miller JF. Evidence for a humoral factor responsible for the maturation of immunologica faculty. Nature $1963 ; 199: 653-4$

9. Guillemin R, Cohn M, Melnechuk T (eds) Neural Modulation of Immunity. New York : Raven Press, 1985.

10. Geenen V, Legros JJ, Franchimont P, et al. The neuroendocrine thymus: coexistence of oxytocin and neurophysin in the human thymus. Science $1986 ; 232: 508-11$.

11. Geenen V, Defresne MP, Robert F, et al. The neurohormonal thymic microenvironment: immunocytochemical evidence that thymic nurse cells are neuroendocrine cells. Neuroendocrinology $1988 ; 47: 365-8$.

12. Martens $\mathrm{H}$, Kecha 0 , Charlet-Renard, C, et al. Neurohypophysial peptides activate phosphorylation of focal adhesion kinases in immature thymocytes. Neuroendocrinology 1998 $67: 282-9$.

13. Hansenne I, Rasier G, Charlet-Renard C, et al. Neurohypophysial receptor gene expression by thymic T cell subsets and thymic T cell lymphoma cell lines. Clin Dev Immunol 2004 ; 11 : $45-51$

14. Ehrlich P. The Croonian lecture: on immunity. Proc Soc Lond Biol 1900; $66: 424$.

15. Burnet FM, Mackay IR. Lymphoepithelial structures and autoimmune disease. The Lancet $1962 ; 2$ : 1030-3.

16. Tonegawa S. Reiteration frequency of immunoglobulin light chain genes: further evidence for somatic generation of antibody diversity. Proc Natl Acad Sci USA 1976 ; 73 : 203-7.

17. Malissen M, Minard K, Mjolsness S, et al. Mouse T cell antigen receptor: structure and organization of constant and joining gene segments encoding the beta polypeptide. Cell $1984 ; 37: 1101-10$.

18. Toyonaga B, Yanagi Y, Suciu-Foca N, et al. Rearrangements of T-cell receptor gene YT35 in human DNA from thymic leukaemia T-cell lines and functional T-cell clones. Nature 1984 ; 311 : 385-7.

19. Davis MM, Chien YH, Gascoigne NR, Hedrick SM. A murine T cell receptor gene complex: isolation, structure and rearrangement. Immunol Rev $1984 ; 81: 235-58$.

20. Ohki H, Martin C, Corbel C, et al. Tolerance induced by thymic epithelial grafts in birds. Science $1987 ; 237: 1032-5$

21. Kappler JW, Roehm N, Marrack P. T cell tolerance by clonal elimination in the thymus. Cell 1987 ; $49: 273-280$.

22. MacDonald HR, Schneider $R$, Lees RK, et al. T-cell receptor $V \beta$ use predict reactivity and tolerance to Mlsa-encoded antigens. Nature $1988 ; 322: 40-5$.

23. Kisielow $P$, Blüthmann $H$, Staerz UD, et al. Tolerance in T-cell receptor transgenic mice involves deletion of nonmature CD4+8+ thymocytes. Nature $1988 ; 333: 742-6$.

24. Wiemann $M$, Ehret $G$. Subcellular characterization of immunoreactive oxytocin within thymic epithelial cells of the male mouse. Cell Tiss Res $1993 ; 273: 573-5$.

25. Funder JW. Paracrine, cryptocrine, acrocrine. Mol Cell Endocrinol $1990 ; 70$ : C21-4.

26. Rammensee HG, Falk K, Rötzschke 0 . Peptides naturally presented by MHC class I molecules. Annu Rev Immunol $1993 ; 11: 213-44$

27. Hashimoto-Tane A, Sakuma M, Ike H, et al. Micro-adhesion rings surrounding TCR microclusters are essential for T cell activation. J Exp Med 2016 ; 213 : 1609-25.

28. Ericsson A, Geenen V, Robert F, et al. Expression of preprotachykinin A and neuropeptide $Y$ messenger RNA in the thymus. Mol Endocrinol $1990 ; 4: 1211-8$.

29. Martens $H$, Goxe B, Geenen V. The thymic repertoire of neuroendocrine self-peptides: physiological implications in T-cell life and death. Immunol Today $1996 ; 17$ : 312-7.

30. Geenen V, Lefèbvre PJ. The intrathymic expression of insulin-related genes: implications for pathophysiology and prevention of type 1 diabetes. Diabetes Metab Rev 1998 ; 14 : 95-103.

31. Vanneste $Y$, Ntodou-Thome A, Vandersmissen $\varepsilon$, et al. Identification of neurotensinrelated peptides in human thymic epithelial cell membranes and relationship with major histocompatibility complex class I molecules. J Neuroimmunol 1997 ; 76 : 161-6.
32. Geenen V, Brilot F, Hansenne $H$, et al. The central role of the thymus in the development of self-tolerance and autoimmunity in the neuroendocrine system. In : Geenen V, Chrousos GP, eds. Immunoendocrinology in Health and Disease. New York: Marcel Dekker, 2004 : 337-355.

33. Geenen V. Presentation of neuroendocrine self in the thymus: a necessity for integrated evolution of the immune and neuroendocrine systems. Ann NY Acad Sci $2012 ; 1261: 42-8$.

34. Geenen V, Bodart G, Henry S, et al. Programming of neuroendocrine self in the thymus and its defect in the development of neuroendocrine autoimmunity. Front Neurosc $2013 ; 7$ : el87.

35. Derbinski J, Schulte A, Kyewski B, Klein L. Promiscuous gene expression in medullary thymic epithelial cells mirrors the peripheral self. Nat Immunol $2001 ; 2: 1032-9$.

36. Kyewski B, Klein L. A central role for central tolerance. Annu Rev Immunol $2006 ; 24: 571-606$

37. Gershon RK, Cohen P, Hencin R, Liebhaber SA. Suppressor T cells. J Immunol $1972 ; 108: 586-90$.

38. Sakagushi S, Sakagushi N, Asano M, et al. Immunologic self-tolerance maintained by activated T cells expressing IL-2 receptor alpha-chain (CD25). Breakdown of a single mechanism of self-tolerance causes various autoimmune diseases. J Immunol 1995 ; 155 : 1151-64.

39. Moulin AM. Le dernier langage de la medicine - Histoire de l'immunologie de Pasteur au SIDA, Paris : Presses Universitaires, 1991.

40. Kecha-Kamoun 0 , Achour I, Martens $\mathrm{H}$, et al. Thymic expression of insulinrelated genes in an animal model of autoimmune type 1 diabetes. Diabetes Metab Rev 2001 ; 17: 146-152.

41. Anderson MS, Venanzi ES, Klein L, et al. Projection of an immunological self shadow within the thymus by the aire protein. Science $2002 ; 298$ : 1395-401.

42. Takaba H, Morishita $Y$, Tomofuji $Y$, et al. Fezf2 orchestrates a thymic program of self-antigen expression for immune tolerance. Cell 2015; 163 : 975-87.

43. Hansenne I, Renard-Charlet C, Greimers R, Geenen V. Dendritic cell differentiation and immune tolerance to insulin-related peptides in Igf2 deficient mice. J Immunol $2006 ; 176: 4651-7$.

44. Brilot F, Chehadeh W, Charlet-Renard C, et al. Persistent infection of human thymic epithelial cells by coxsackievirus B4. J Virol $2002 ; 76$ 5260-5.

45. Jaïdane $\mathrm{H}$, Caloone D, Lobert PE, et al. Persistent infection of thymic epithelial cells with coxsackievirus B4 results in decreased expression of type 2 insulin-like growth factor. J Virol $201 ; 86$ : 11151-62.

46. Paschke R, Geenen, V. Messenger RNA expression for a TSH receptor variant in the thymus of a two-year old child. J Mol Med $1995 ; 73$ : 577-80.

47. Murakami M, Hosoi Y, Negishi T, et al. (1996). Thymic hyperplasia in patients with Graves' disease. Identification of thyrotropin receptors in human thymus. . Clin Invest $1996 ; 98: 2228-34$.

48. Geenen V, Mottet M, Dardenne 0, et al. Thymic self-antigens for the design of a negative/tolerogenic self-vaccination against type 1 diabetes. Curr Opin Pharmacol $2010 ; 10: 461-72$.

49. Bajoghli B, Guo P, Aghaallaei N, et al. A thymus candidate in lampreys. Nature $2011 ; 470: 90-4$

50. Lopes N, Ferrier P, Irla M. Induction de la tolérance centrale dans le thymus par le facteur de transcription Aire. Med Sci (Paris) 2015 ; 31 : $742-7$

51. Dragin N, Le Panse R, Berrih-Aknin S. Prédisposition aux pathologies autoimmmunes : les hommes ne manquent pas «d'Aire ». Med Sci (Paris) 2017 $33: 169-75$.

\section{Également à consulte}

52. Tauber Al. The immune self: theory or metaphor? Immunol Today 1994 ; $15: 134-6$.

53. Silverstein AM. A history of immunology, $2^{\text {nd }}$ edition. London : Academic Press, 2009. 